Proyecciones Journal of Mathematics

Vol. 34, No 3, pp. 229-241, September 2015.

Universidad Católica del Norte

Antofagasta - Chile

\title{
Stability in delay Volterra difference equations of neutral type
}

\author{
Ernest Yankson \\ and \\ Emmanuel K. Essel \\ University of Cape Coast, Ghana \\ Received: March 2015. Accepted : June 2015
}

\begin{abstract}
Sufficient conditions for the zero solution of a certain class of neutral Volterra difference equations with variable delays to be asymptotically stable are obtained. The Banach's fixed point theorem is employed in proving our results.
\end{abstract}

2000 Mathematics Subject Classification: 349A30, 39A70.

Keywords : Banach's Fixed point theorem, Volterra difference equation, asymptotic stability. 


\section{Introduction}

The study of the stability of the zero solution of difference equations has gained the attention of many mathematicians lately, see [1], [2], [3], [5], [7], [9], [11] and [12]. In this paper we consider the nonlinear difference equation with variable delays

$$
\begin{aligned}
\Delta x(n)= & -\sum_{j=1}^{N} a_{j}(n) x\left(n-\tau_{j}(n)\right)+\sum_{j=1}^{N} \Delta Q_{j}\left(n, x\left(n-\tau_{j}(n)\right)\right) \\
& +\sum_{j=1}^{N} \sum_{s=n-\tau_{j}(n)}^{n-1} k_{j}(n, s) f_{j}(s, x(s)),
\end{aligned}
$$

with the initial condition

$$
x(n)=\psi(n) \text { for } n \in\left[m\left(n_{0}\right), n_{0}\right] \cap \mathbf{Z},
$$

where $\psi:\left[m\left(n_{0}\right), n_{0}\right] \cap \mathbf{Z} \rightarrow \mathbf{R}$ is a bounded sequence and for $n_{0} \geq 0$,

$m_{j}\left(n_{0}\right)=\inf \left\{n-\tau_{j}(n), n \geq n_{0}\right\}, m\left(n_{0}\right)=\min \left\{m_{j}\left(n_{0}\right), 1 \leq j \leq N\right\}$.

Here $\Delta$ denotes the forward difference operator. That is, $\Delta x(n)=x(n+$ $1)-x(n)$ for any sequence $\left\{x(n): n \in \mathbf{Z}^{+}\right\}$. We assume throughout this paper that $a_{j}: \mathbf{Z}^{+} \rightarrow \mathbf{R}, k_{j}: \mathbf{Z}^{+} \times\left(\left[m_{j}\left(n_{0}\right), \infty\right) \cap \mathbf{Z}\right) \rightarrow \mathbf{R}, f_{j}: \mathbf{Z}^{+} \times \mathbf{R} \rightarrow \mathbf{R}$, $Q_{j}: \mathbf{Z}^{+} \times \mathbf{R} \rightarrow \mathbf{R}$ and $\tau_{j}: \mathbf{Z}^{+} \rightarrow \mathbf{Z}^{+}$, for $j=1, \ldots, N$. Special cases of (1.1) have been considered by a number of researchers in recent times.

For instance, Raffoul in [7] considered the equation

$$
\Delta x(n)=-a(n) x(n-\tau)
$$

where $\tau$ is a positive constant. The first author in [11], extended the results obtained in [7] to the equation

$$
\Delta x(n)=-\sum_{j=1}^{N} a_{j}(n) x\left(n-\tau_{j}(n)\right) .
$$


The first author also in [12] considered the the following nonlinear Volterra difference equation

$$
x(n+1)=a(n) x(n)+c(n) \Delta x(n-\tau(n))+\sum_{s=n-\tau(n)}^{n-1} k(n, s) q(x(s)) .
$$

Ardjouni and Djoudi in [1] considered the nonlinear Volterra difference equation with variable delays

$x(n+1)=a(n) x\left(n-\tau_{1}(n)\right)+c(n) \Delta x\left(n-\tau_{2}(n)\right)+\sum_{s=n-\tau_{2}(n)}^{n-1} k(n, s) q(x(s))$.

Moreover, Ardjouni and Djoudi in [2] considered the difference equations with variable delays

$$
\Delta x(n)=-\sum_{j=1}^{N} a_{j}(n) x\left(n-\tau_{j}(n)\right)+\sum_{j=1}^{N} c_{j}(n) \Delta x\left(n-\tau_{j}(n)\right)
$$

Motivated by the above mentioned papers, we obtain in this paper sufficient conditions for the zero solution of (1.1) to be asymptotically stable.

\section{Stability}

Let $n_{0} \in \mathbf{Z} \cap[0, \infty)$, be fixed. We let $D\left(n_{0}\right)$ be the set of bounded sequences $\psi:\left[m\left(n_{0}\right), n_{0}\right] \cap \mathbf{Z} \rightarrow \mathbf{R}$, with the norm $|\psi|_{0}=\max \{|\psi(n)|: n \in$ $\left.\left[m\left(n_{0}\right), n_{0}\right] \cap \mathbf{Z}\right\}$. Also, let $(\mathbf{B},\|\|$.$) be the Banach space of bounded se-$ quences $x:\left[m\left(n_{0}\right), \infty\right) \cap \mathbf{Z} \rightarrow \mathbf{R}$ with the maximum norm $\|$.$\| .$

In this paper we assume that for $\mathrm{j}=1, \ldots, \mathrm{N}$,

$$
\left|Q_{j}(n, x)-Q_{j}(n, y)\right| \leq L_{1}\|x-y\|,
$$

and

$$
\left|f_{j}(n, x)-f_{j}(n, y)\right| \leq L_{2}|| x-y||
$$

for some positive constants $L_{1}$ and $L_{2}$. Also, for $\mathrm{j}=1, \ldots, \mathrm{N}$, 


$$
f_{j}(n, 0)=0, \quad Q_{j}(n, 0)=0,
$$

and

$$
n-\tau_{j}(n) \rightarrow \infty \text { as } n \rightarrow \infty
$$

Lemma 2.1 Let $h_{j}:\left[m\left(n_{0}\right), \infty\right) \cap \mathbf{Z} \rightarrow \mathbf{R}$ be an arbitrary sequence, for $j=$ $1, \ldots, N$. Suppose that $H(n)=1-\sum_{j=1}^{N} h_{j}(n) \neq 0$, for all $n \in\left[n_{o}, \infty\right) \cap \mathbf{Z}$. Then $x$ is a solution of equation (1.1) if and only if

$$
\begin{aligned}
x(n)= & {\left[x\left(n_{0}\right)-\sum_{j=1}^{N} Q_{j}\left(n_{0}, x\left(n_{0}-\tau_{j}\left(n_{0}\right)\right)\right)-\sum_{j=1}^{N} \sum_{s=n_{0}-\tau_{j}\left(n_{0}\right)}^{n_{0}-1} h_{j}(s) x(s)\right] } \\
& \prod_{u=n_{0}}^{n-1} H(u) \\
& +\sum_{j=1}^{N} Q_{j}\left(n, x\left(n-\tau_{j}(n)\right)\right)+\sum_{j=1}^{N} \sum_{s=n-\tau_{j}(n)}^{n-1} h_{j}(s) x(s) \\
& +\sum_{s=n_{0}}^{n-1}\left[\sum_{j=1}^{N}\left\{h_{j}\left(n-\tau_{j}(n)\right)-a_{j}(n)\right\} x\left(n-\tau_{j}(n)\right)\right. \\
& -[1-H(s)] \sum_{j=1}^{N} Q_{j}\left(s, x\left(s-\tau_{j}(s)\right)\right)-[1-H(s)] \\
& \sum_{j=1}^{N} \sum_{r=s-\tau_{j}(s)}^{s-1} h_{j}(r) x(r) \\
& \left.+\sum_{j=1}^{N} \sum_{u=s-\tau_{j}(s)}^{s-1} k_{j}(s, u) f_{j}(u, x(u))\right] \prod_{u=s+1}^{n-1} H(u) .
\end{aligned}
$$

Proof. Rewrite (1.1) as

$$
\Delta x(n)=-\sum_{j=1}^{N} h_{j}(n) x(n)+\Delta_{n} \sum_{j=1}^{N} \sum_{s=n-\tau_{j}(n)}^{n-1} h_{j}(s) x(s)
$$




$$
\begin{aligned}
& +\sum_{j=1}^{N}\left\{h_{j}\left(n-\tau_{j}(n)\right)-a_{j}(n)\right\} x\left(n-\tau_{j}(n)\right) \\
& +\sum_{j=1}^{N} \Delta Q_{j}\left(n, x\left(n-\tau_{j}(n)\right)\right) \\
& +\sum_{j=1}^{N} \sum_{s=n-\tau_{j}(n)}^{n-1} k_{j}(n, s) f_{j}(s, x(s)),
\end{aligned}
$$

where $\Delta_{n}$ denotes the difference taken with respect to $n$.

The above equation is equivalent to

$$
\begin{aligned}
x(n+1)= & H(n) x(n)+\Delta_{n} \sum_{j=1}^{N} \sum_{s=n-\tau_{j}(n)}^{n-1} h_{j}(s) x(s) \\
& +\sum_{j=1}^{N}\left\{h_{j}\left(n-\tau_{j}(n)\right)-a_{j}(n)\right\} x\left(n-\tau_{j}(n)\right) \\
& +\sum_{j=1}^{N} \Delta Q_{j}\left(n, x\left(n-\tau_{j}(n)\right)\right) \\
& +\sum_{j=1}^{N} \sum_{s=n-\tau_{j}(n)}^{n-1} k_{j}(n, s) f_{j}(s, x(s)) .
\end{aligned}
$$

Rewrite equation (2.6) as

$$
\begin{aligned}
\Delta_{n}\left[\prod_{u=n_{0}}^{n-1} H(u)^{-1} x(u)\right]= & {\left[\Delta_{n} \sum_{j=1}^{N} \sum_{s=n-\tau_{j}(n)}^{n-1} h_{j}(s) x(s)\right.} \\
& +\sum_{j=1}^{N}\left\{h_{j}\left(n-\tau_{j}(n)\right)-a_{j}(n)\right\} x\left(n-\tau_{j}(n)\right) \\
& +\sum_{j=1}^{N} \Delta Q_{j}\left(n, x\left(n-\tau_{j}(n)\right)\right)
\end{aligned}
$$

$$
\left.+\sum_{j=1}^{N} \sum_{s=n-\tau_{j}(n)}^{n-1} k_{j}(n, s) f_{j}(s, x(s))\right] \prod_{u=n_{0}}^{n} H(u)^{-1} .
$$


Summing (2.7) from $n_{0}$ to $n-1$ we obtain

$$
\begin{aligned}
\sum_{s=n_{0}}^{n-1} \Delta_{s}\left[\prod_{u=n_{0}}^{s-1} H(u)^{-1} x(s)\right]= & \sum_{s=n_{0}}^{n-1}\left[\Delta_{s} \sum_{j=1}^{N} \sum_{r=s-\tau_{j}(s)}^{s-1} h_{j}(r) x(r)\right. \\
& +\sum_{j=1}^{N}\left\{h_{j}\left(n-\tau_{j}(n)\right)-a_{j}(n)\right\} x\left(n-\tau_{j}(n)\right) \\
& +\sum_{j=1}^{N} \Delta Q_{j}\left(n, x\left(n-\tau_{j}(n)\right)\right) \\
& \left.+\sum_{j=1}^{N} \sum_{u=s-\tau_{j}(s)}^{s-1} k_{j}(s, u) f_{j}(u, x(u))\right] \prod_{u=n_{0}}^{s} H(u)^{-1} .
\end{aligned}
$$

Consequently, we have

$$
\begin{aligned}
& \prod_{u=n_{0}}^{n-1} H(u)^{-1} x(n)-\prod_{u=n_{0}}^{n_{0}-1} H(u)^{-1} x\left(n_{0}\right) \\
& =\sum_{s=n_{0}}^{n-1}\left[\Delta_{s} \sum_{j=1}^{N} \sum_{r=s-\tau_{j}(s)}^{s-1} h_{j}(r) x(r)\right. \\
& +\sum_{j=1}^{N}\left\{h_{j}\left(n-\tau_{j}(n)\right)-a_{j}(n)\right\} x\left(n-\tau_{j}(n)\right) \\
& +\sum_{j=1}^{N} \Delta Q_{j}\left(n, x\left(n-\tau_{j}(n)\right)\right) \\
& \left.+\sum_{j=1}^{N} \sum_{u=s-\tau_{j}(s)}^{s-1} k_{j}(s, u) f_{j}(u, x(u))\right] \prod_{u=n_{0}}^{s} H(u)^{-1} .
\end{aligned}
$$

Dividing both sides of (2.8) by $\prod_{u=n_{0}}^{n-1} H(u)^{-1}$ we obtain

$$
\begin{aligned}
x(n)= & x\left(n_{0}\right) \prod_{u=n_{0}}^{n-1} H(u)+\sum_{s=n_{0}}^{n-1}\left[\Delta_{s} \sum_{j=1}^{N} \sum_{r=s-\tau_{j}(s)}^{s-1} h_{j}(r) x(r)\right. \\
& +\sum_{j=1}^{N}\left\{h_{j}\left(n-\tau_{j}(n)\right)-a_{j}(n)\right\} x\left(n-\tau_{j}(n)\right) \\
& +\sum_{j=1}^{N} \Delta Q_{j}\left(n, x\left(n-\tau_{j}(n)\right)\right)
\end{aligned}
$$




$$
\left.+\sum_{j=1}^{N} \sum_{u=s-\tau_{j}(s)}^{s-1} k_{j}(s, u) f_{j}(u, x(u))\right] \prod_{u=s+1}^{n-1} H(u) .
$$

Using the summation by parts formula, we obtain

$$
\begin{aligned}
& \sum_{s=n_{0}}^{n-1} \prod_{u=s+1}^{n-1} H(u)\left[\sum_{j=1}^{N} \Delta Q_{j}\left(n, x\left(n-\tau_{j}(n)\right)\right)\right] \\
& =\sum_{j=1}^{N} Q_{j}\left(n, x\left(n-\tau_{j}(n)\right)\right)-\sum_{j=1}^{N} Q_{j}\left(n_{0}, x\left(n_{0}-\tau_{j}\left(n_{0}\right)\right)\right) \prod_{u=n_{0}}^{n-1} H(u) \\
& -\sum_{s=n_{0}}^{n-1} \sum_{j=1}^{N} Q_{j}\left(s, x\left(s-\tau_{j}(s)\right)\right)[1-H(s)] \prod_{u=s+1}^{n-1} H(u),
\end{aligned}
$$

and

$$
\begin{aligned}
\sum_{s=n_{0}}^{n-1} \prod_{u=s+1}^{n-1} H(u)\left[\Delta_{s} \sum_{j=1}^{N} \sum_{r=s-\tau_{j}(s)}^{s-1} h_{j}(r) x(r)\right] \\
=\sum_{j=1}^{N} \sum_{s=n-\tau_{j}(n)}^{n-1} h_{j}(s) x(s)-\prod_{u=n_{0}}^{n-1} H(u) \sum_{j=1}^{N} \sum_{s=n_{0}-\tau_{j}\left(n_{0}\right)}^{n_{0}-1} h_{j}(s) x(s) \\
-\sum_{s=n_{0}}^{n-1}[1-H(s)] \prod_{u=s+1}^{n-1} H(u) \sum_{j=1}^{N} \sum_{r=s-\tau_{j}(s)}^{s-1} h_{j}(r) x(r) .
\end{aligned}
$$

Substituting (2.10) and (2.11) into (2.9) gives the desired results.

Theorem 2.1 Suppose (2.1), (2.2), (2.3) and (2.4) hold and let $h_{j}$ : $\left[m\left(n_{0}\right), \infty\right) \cap \mathbf{Z} \rightarrow \mathbf{R}$ be an arbitrary sequence, for $j=1, \ldots, N$, such that $H(n)=1-\sum_{j=1}^{N} h_{j}(n) \neq 0$, for all $n \in\left[n_{o}, \infty\right) \cap \mathbf{Z}$. Suppose further that there exist a constant $\alpha \in(0,1)$ such that

$$
\begin{aligned}
& N L_{1}+\sum_{j=1}^{N} \sum_{s=n-\tau_{j}(n)}^{n-1}\left|h_{j}(s)\right| \\
& +\sum_{s=n_{0}}^{n-1}\left[\sum_{j=1}^{N}\left|h_{j}\left(n-\tau_{j}(n)\right)-a_{j}(n)\right|\right.
\end{aligned}
$$




$$
\begin{aligned}
& +|1-H(s)| N L_{1}+|1-H(s)| \sum_{j=1}^{N} \sum_{r=s-\tau_{j}(s)}^{s-1}\left|h_{j}(r)\right| \\
& \left.+L_{2} \sum_{j=1}^{N} \sum_{u=s-\tau_{j}(s)}^{s-1}\left|k_{j}(s, u)\right|\right]\left|\prod_{u=s+1}^{n-1} H(u)\right| \leq \alpha .
\end{aligned}
$$

Moreover, assume that there exist a positive constant $G$ such that

$$
\left|\prod_{u=n_{0}}^{n-1} H(u)\right| \leq G
$$

and

$$
\prod_{u=n_{0}}^{n-1} H(u) \rightarrow 0 \text { as } n \rightarrow \infty .
$$

Then the zero solution of (1.1) is asymptotically stable.

Proof. Let $\epsilon>0$ be given. Choose $\delta>0$ such that

$$
\delta G[1+\alpha]+\epsilon \alpha \leq \epsilon .
$$

Let $\psi \in D\left(n_{0}\right)$ such that $|\psi(n)| \leq \delta$ and define

$$
\begin{gathered}
S=\left\{\varphi \in B: \varphi(n)=\psi(n) \text { if } n \in\left[m\left(n_{0}\right), n_{0}\right] \cap \mathbf{Z}\right. \\
\|\varphi\| \leq \epsilon \text { and } \varphi(n) \rightarrow 0 \text { as } n \rightarrow \infty\} .
\end{gathered}
$$

Then $(S,\|\|$.$) is a complete metric space where, \|$.$\| is the maximum norm.$

Define the mapping $P: S \rightarrow S$ by

$$
(P \varphi)(n)=\psi(n) \text { for } n \in\left[m\left(n_{0}\right), n_{0}\right] \cap \mathbf{Z},
$$

and

$$
\begin{aligned}
& (P \varphi)(n)=\left[\psi\left(n_{0}\right)-\sum_{j=1}^{N} Q_{j}\left(n_{0}, \psi\left(n_{0}-\tau_{j}\left(n_{0}\right)\right)\right)-\sum_{j=1}^{N} \sum_{s=n_{0}-\tau_{j}\left(n_{0}\right)}^{n_{0}-1} h_{j}(s) \psi(s)\right] \\
& \prod_{u=n_{0}}^{n-1} H(u)
\end{aligned}
$$




$$
\begin{aligned}
& +\sum_{j=1}^{N} Q_{j}\left(n, \varphi\left(n-\tau_{j}(n)\right)\right)+\sum_{j=1}^{N} \sum_{s=n-\tau_{j}(n)}^{n-1} h_{j}(s) \varphi(s) \\
& +\sum_{s=n_{0}}^{n-1}\left[\sum_{j=1}^{N}\left\{h_{j}\left(n-\tau_{j}(n)\right)-a_{j}(n)\right\} \varphi\left(n-\tau_{j}(n)\right)\right. \\
& -[1-H(s)] \sum_{j=1}^{N} Q_{j}\left(s, \varphi\left(s-\tau_{j}(s)\right)\right)-[1-H(s)] \sum_{j=1}^{N} \sum_{r=s-\tau_{j}(s)}^{s-1} h_{j}(r) \varphi(r) \\
& \left.+\sum_{j=1}^{N} \sum_{u=s-\tau_{j}(s)}^{s-1} k_{j}(s, u) f_{j}(u, \varphi(u))\right] \prod_{u=s+1}^{n-1} H(u), n \geq n_{0} .
\end{aligned}
$$

Clearly, $P \varphi$ is continuous. We first show that $P: S \rightarrow S$. Using (2.15) we obtain

$$
\begin{aligned}
|(P \varphi)(n)| \leq & \delta G[1+\alpha]+\left\{N L_{1}+\sum_{j=1}^{N} \sum_{s=n-\tau_{j}(n)}^{n-1}\left|h_{j}(s)\right|\right. \\
& +\sum_{s=n_{0}}^{n-1}\left[\sum_{j=1}^{N}\left|h_{j}\left(n-\tau_{j}(n)\right)-a_{j}(n)\right|\right. \\
& +|1-H(s)| N L_{1}+|1-H(s)| \sum_{j=1}^{N} \sum_{r=s-\tau_{j}(s)}^{s-1}\left|h_{j}(r)\right| \\
& \left.\left.+L_{2} \sum_{j=1}^{N} \sum_{u=s-\tau_{j}(s)}^{s-1}\left|k_{j}(s, u)\right|\right]\left|\prod_{u=s+1}^{n-1} H(u)\right|\right\}|| \varphi|| \\
\leq & \delta G[1+\alpha]+\alpha \epsilon \\
\leq & \epsilon .
\end{aligned}
$$

We next show that $(P \varphi)(n) \rightarrow 0$ as $n \rightarrow \infty$. The first term on the right hand side of (2.15) goes to zero in view of condition (2.14). Since $\varphi(n) \rightarrow 0$ and $n-\tau_{j}(n) \rightarrow \infty$ as $n \rightarrow \infty$, we have that $Q_{j}\left(n, \varphi\left(n-\tau_{j}(n)\right)\right) \rightarrow$ $Q_{j}(n, 0)=0$ as $n \rightarrow \infty$ for $j=1, \ldots, N$. Thus showing that the second term on the right hand side of (2.15) goes to zero as $n \rightarrow \infty$. 
Let $\varphi \in S$ be fixed. The fact that $\varphi(n) \rightarrow 0$ and $n-\tau_{j}(n) \rightarrow \infty$ as $n \rightarrow \infty$, implies that, given $\epsilon_{1}>0$ there exists $N_{1}>n-\tau_{j}(n)$ for $j=1, \ldots, N$ such that $|\varphi(s)| \leq \epsilon_{1}$ for $s \geq N_{1}$. Thus

$$
\begin{aligned}
\left|\sum_{j=1}^{N} \sum_{s=n-\tau_{j}(n)}^{n-1} h_{j}(s) \varphi(s)\right| & \leq \epsilon_{1} \sum_{j=1}^{N} \sum_{s=n-\tau_{j}(n)}^{n-1}\left|h_{j}(s)\right| \\
& \leq \alpha \epsilon_{1}<\epsilon_{1} .
\end{aligned}
$$

Thus showing that the third term on the right hand side of (2.15) goes to zero as $n \rightarrow \infty$. We next show that the last term on the right hand side of (2.15) goes to zero as $n \rightarrow \infty$. Since $\varphi(n) \rightarrow 0$ and $n-\tau_{j}(n) \rightarrow \infty$ as $n \rightarrow \infty$, for each $\epsilon_{2}>0$, there exists $N_{2}>n_{0}$ such that $s \geq N_{2}$ implies $\left|\varphi\left(s-\tau_{j}(s)\right)\right|<\epsilon_{2}$ for $j=1, \ldots, N$. Thus for $n \geq N_{2}$, the last term on the right hand side of (2.15) satisfies

$$
\begin{aligned}
& \mid \sum_{s=n_{0}}^{n-1}\left[\sum_{j=1}^{N}\left\{h_{j}\left(n-\tau_{j}(n)\right)-a_{j}(n)\right\} \varphi\left(n-\tau_{j}(n)\right)\right. \\
& -[1-H(s)] \sum_{j=1}^{N} Q_{j}\left(s, \varphi\left(s-\tau_{j}(s)\right)\right)-[1-H(s)] \sum_{j=1}^{N} \sum_{r=s-\tau_{j}(s)}^{s-1} h_{j}(r) \varphi(r) \\
& \left.+\sum_{j=1}^{N} \sum_{u=s-\tau_{j}(s)}^{s-1} k_{j}(s, u) f_{j}(u, \varphi(u))\right] \prod_{u=s+1}^{n-1} H(u) \mid \\
& \leq \sum_{s=n_{0}}^{N_{2}-1}\left[\sum_{j=1}^{N}\left|h_{j}\left(s-\tau_{j}(s)\right)-a_{j}(s)\right|\left|\varphi\left(s-\tau_{j}(s)\right)\right|\right. \\
& +|1-H(s)| L_{1} \sum_{j=1}^{N}\left|\varphi\left(s-\tau_{j}(s)\right)\right|+|1-H(s)| \sum_{j=1}^{N} \sum_{r=s-\tau_{j}(s)}^{s-1}\left|h_{j}(r)\right||\varphi(r)| \\
& \left.+L_{2} \sum_{j=1}^{N} \sum_{u=s-\tau_{j}(s)}^{s-1}\left|k_{j}(s, u)\right||\varphi(u)|\right]\left|\prod_{u=s+1}^{n-1} H(u)\right| \\
& +\sum_{s=N_{2}}^{n-1}\left[\sum_{j=1}^{N}\left|h_{j}\left(s-\tau_{j}(s)\right)-a_{j}(s)\right|\left|\varphi\left(s-\tau_{j}(s)\right)\right|\right. \\
& +|1-H(s)| L_{1} \sum_{j=1}^{N}\left|\varphi\left(s-\tau_{j}(s)\right)\right|+|1-H(s)| \sum_{j=1}^{N} \sum_{r=s-\tau_{j}(s)}^{s-1}\left|h_{j}(r)\right||\varphi(r)|
\end{aligned}
$$




$$
\begin{aligned}
& \left.+L_{2} \sum_{j=1}^{N} \sum_{u=s-\tau_{j}(s)}^{s-1}\left|k_{j}(s, u)\right||\varphi(u)|\right]\left|\prod_{u=s+1}^{n-1} H(u)\right| \\
& \leq \max _{\sigma \geq m\left(n_{0}\right)} \varphi(\sigma) \sum_{s=n_{0}}^{N_{2}-1}\left[\sum_{j=1}^{N}\left|h_{j}\left(s-\tau_{j}(s)\right)-a_{j}(s)\right|\right. \\
& +|1-H(s)| L_{1} N+|1-H(s)| \sum_{j=1}^{N} \sum_{r=s-\tau_{j}(s)}^{s-1}\left|h_{j}(r)\right| \\
& \left.+L_{2} \sum_{j=1}^{N} \sum_{u=s-\tau_{j}(s)}^{s-1}\left|k_{j}(s, u)\right|\right]\left|\prod_{u=s+1}^{n-1} H(u)\right| \\
& +\epsilon_{2} \sum_{s=N_{2}}^{n-1}\left[\sum_{j=1}^{N}\left|h_{j}\left(s-\tau_{j}(s)\right)-a_{j}(s)\right|\right. \\
& +|1-H(s)| L_{1} N+|1-H(s)| \sum_{j=1}^{N} \sum_{r=s-\tau_{j}(s)}^{s-1}\left|h_{j}(r)\right| \\
& \left.+L_{2} \sum_{j=1}^{N} \sum_{u=s-\tau_{j}(s)}^{s-1}\left|k_{j}(s, u)\right|\right]\left|\prod_{u=s+1}^{n-1} H(u)\right| \\
& \leq \epsilon_{2}+\epsilon_{2} \alpha<2 \epsilon_{2} .
\end{aligned}
$$

Thus showing that the last term on the right hand side of (2.15) goes to zero as $n \rightarrow \infty$. Therefore, $(P \varphi) \rightarrow 0$ as $n \rightarrow \infty$. It therefore follows that $P$ maps $S$ into $S$.

We finally show that $P$ is a contraction. Let $\varphi, \eta \in S$. Then

$$
\begin{aligned}
|(P \varphi)(n)-(P \eta)(n)| \leq & \left\{N L_{1}+\sum_{j=1}^{N} \sum_{s=n-\tau_{j}(n)}^{n-1}\left|h_{j}(s)\right|\right. \\
& +\sum_{s=n_{0}}^{n-1}\left[\sum_{j=1}^{N}\left|h_{j}\left(n-\tau_{j}(n)\right)-a_{j}(n)\right|\right. \\
& +|1-H(s)| N L_{1}+|1-H(s)| \sum_{j=1}^{N} \sum_{r=s-\tau_{j}(s)}^{s-1}\left|h_{j}(r)\right| \\
& \left.\left.+L_{2} \sum_{j=1}^{N} \sum_{u=s-\tau_{j}(s)}^{s-1}\left|k_{j}(s, u)\right|\right]\left|\prod_{u=s+1}^{n-1} H(u)\right|\right\}|| \varphi-\eta \| \\
\leq \alpha|\varphi-\eta| \mid . &
\end{aligned}
$$


This shows that $P$ is a contraction. Therefore, by the contraction mapping principle, $P$ has a unique fixed point in $S$ which solves (1.1) and for any $\varphi \in S,\|P \varphi\| \leq \epsilon$. This shows that the zero solution of (1.1) is stable. Moreover, $(P \varphi) \rightarrow 0$ as $n \rightarrow \infty$, showing that the zero solution of (1.1) is asymptotically stable. This completes the proof.

\section{References}

[1] A. Ardjouni, and A. Djoudi; Stability in nonlinear neutral Volterra difference equations with variable delays, Journal of Nonlinear Evolution Equations and Applications, No. 7, pp. 89-100, (2013).

[2] A. Ardjouni, and A. Djoudi; Stability in linear neutral difference equations with variable delays, Mathematica Bohemica, No. 3, pp. 245-258, (2013).

[3] S. Elaydi, Periodicity and stability of linear Volterra difference systems, Journal of Mathematical Analysis and Applications, 181, pp. 483-492, (1994).

[4] S. Elaydi, An Introduction to Difference Equations, Springer, New York, (1999).

[5] M. Islam and E. Yankson, Boundedness and stability in nonlinear delay difference equations employing fixed point theory, Electronic Journal of Qualitative Theory of Differential Equations, No. 26, pp. 1-18, (2005).

[6] W. G. Kelly and A. C. Peterson, Difference Equations : An Introduction with Applications, Academic Press, (2001).

[7] Y. N. Raffoul, Stability and periodicity in discrete delay equations, Journal of Mathematical Analysis and Applications, 324, No. 2, pp. 1356-1362, (2006).

[8] Y. N. Raffoul, Periodicity in general delay nonlinear difference equations using fixed point theory, Journal of Difference Equations and Applications, 10, No. 1315, pp. 1229-1242, (2004).

[9] Y. N. Raffoul, General theorems for stability and boundedness for nonlinear functional discrete systems, Journal of Mathematical Analysis and Applications, 279, pp. 639-650, (2003). 
[10] D. R. Smart, Fixed point theorems ; Cambridge Tracts in Mathematics, No. 66. Cambridge University Press, London-New York, (1974).

[11] E. Yankson, Stability in discrete equations with variable delays, Electronic Journal of Qualitative Theory of Differential Equations, No. 8, pp. 1-7, (2009).

[12] E. Yankson, Stability of Volterra difference delay equations, Electronic Journal of Qualitative Theory of Differential Equations, No. 20, pp. 1-14, (2006).

\section{Ernest Yankson}

Department of Mathematics and Statistics

University of Cape Coast

Ghana

e-mail : ernestoyank@gmail.com

and

\section{Emmanuel K. Essel}

Department of Mathematics and Statistics

University of Cape Coast

Ghana

e-mail : ekessel04@yahoo.co.uk 